\title{
Diagnostic value of triple-phase bone scintigraphy for the diagnosis of infection around antibiotic-impregnated cement spacers
}

\author{
Masahiko Ikeuchi ${ }^{1{ }^{*}}$, Yusuke Okanoue ${ }^{1+}$, Masashi Izumi ${ }^{1}$, Goichi Fukuda ${ }^{1}$, Koji Aso ${ }^{1}$, Natsuki Sugimura ${ }^{1}$,
}

Teruhiko Kawakami ${ }^{2}$ and Toshikazu Tani ${ }^{1}$

\begin{abstract}
Introduction: Two-stage revision arthroplasty is today considered as the gold standard for control of chronic deep prosthetic infection. Although the revision arthroplasty should only be considered when infection is eliminated, the diagnosis of residual infection prior to the revision remains a challenging problem.

Materials and methods: We evaluated triple-phase bone scintigraphy as a useful diagnostic test for the detection of residual infection around the antibiotic-impregnated cement spacer in patients waiting for the second-stage revision hip or knee arthroplasty. Increased uptake of radioisotope in all three phases was considered positive for infection. The final diagnosis was based on histopathological results in addition to microbiologic examinations of surgical specimens.

Results: Histopathological examination showed positive in 17 and negative in 13 out of 30 examinations. Among 17 samples positive for histopathology, there were only 4 samples positive for bacterial culture. All samples negative for histopathology showed negative for bacterial culture. The positive and negative predictive values of triple-phase bone scintigraphy for the presence of infection were $80 \%$ and $90 \%$, respectively. The diagnostic sensitivity was $94 \%$ and the specificity was $69 \%$.

Conclusion: Triple-phase bone scintigraphy was useful in the diagnosis of infection around the articulating cement spacer. In particular, when triple-phase bone scintigraphy shows negative, the residual infection around the cement spacer is unlikely.
\end{abstract}

Keywords: Knee; Hip; Arthroplasty; Infection; Revision; Triple-phase bone scintigraphy

\section{Introduction}

Periprosthetic infection occurs in less than $2 \%$ of patients (Phillips et al. 2006; Pedersen et al. 2010; Garvin and Konigsberg 2011) but nevertheless is one of the most devastating complications following hip and knee arthroplasties. Treatment options for periprothetic infection include irrigation and debridement (Van Kleunen et al. 2010), direct exchange arthroplasty (Ure et al. 1998; Silva et al. 2002), and two-stage revision arthroplasty (Biring et al. 2009; Borowski et al. 2012; Mahmud et al. 2012).

\footnotetext{
* Correspondence: ikeuchim@kochi-u.ac.jp

${ }^{\dagger}$ Equal contributors

${ }^{1}$ Department of Orthopaedic Surgery, Kochi Medical School, Kochi University, Kohasu, Oko-cho, Nankoku, Kochi 783-8505, Japan

Full list of author information is available at the end of the article
}

Two-stage revision arthroplasty is today considered as the gold standard for control of chronic deep prosthetic infection (Cui et al. 2007; Sukeik and Haddad 2009; Mahmud et al. 2012). The procedure consists of implant removal, thorough debridement, placement of an antibiotic-impregnated cement spacer, a course of intravenous antibiotics, and a delayed second-stage revision arthroplasty (Cui et al. 2007).

The second-stage revision arthroplasty should only be considered when no signs of residual infection are present. Common investigations prior to the secondstage revision include serologic tests such as white blood cell count, erythrocyte sedimentation rate (ESR), and C-reactive protein (CRP), and joint fluid aspiration. However, normal serologic tests and negative cultures 
do not guarantee eradication of infection (Tunney et al. 1999; Bare et al. 2006; Bauer et al. 2006; Berbari et al. 2010). In particular, low-grade infection increases falsenegative results (Sanzen and Sundberg 1997). Hence, the diagnosis of residual infection prior to the second-stage revision remains a challenging problem and requires additional diagnostic tests.

Triple-phase bone scintigraphy (TPBS) has been used to diagnose osteomyelitis (Schauwecker 1992) and periprosthetic infection (Rubello et al. 1995; Stumpe et al. 2004; Reinartz et al. 2005; Nagoya et al. 2008). When isotope uptake is increased in all three phases, periprosthetic infection can be differentiated from aseptic mechanical loosening with $80 \%$ accuracy for the hip and $81 \%$ accuracy for the knee (Stumpe et al. 2004; Nagoya et al. 2008; Reinartz 2009). The articulating cement spacer (Fehring et al. 2000; Biring et al. 2009) recently used during the waiting period is considered equivalent to a loose prosthetic component, once the postoperative acute phase is past. Therefore, it was expected that the TPBS would be useful in the diagnosis of infection around the articulating cement spacer. We have used the TPBS for this purpose since 2005. The aim of this retrospective study was to evaluate the TPBS as a useful diagnostic test for the detection of residual infection around the articulating cement spacer.

\section{Patients and methods}

From January 2005 to December 2009, the TPBS followed by histopathological examination was performed 30 times in consecutive 15 patients who were treated for chronic deep periprosthetic infection at our institution. There were 4 men and 11 women, with a mean age at first operation 68 years (range 47-85). Infections occurred after primary total hip arthroplasty (THA) in 10 patients and after primary total knee arthroplasty (TKA) in 5 patients. Methicillin-resistant Staphylococcus aureus was detected in 5 patients, Methicillin-resistant Staphylococcus epider midis in 2, Staphylococcus aureus in 3, Methicillinresistant Staphylococcus capitis in 1, Staphylococcus capitis in 1 , and undetected in 3 . Three patients with undetected microorganism were diagnosed with periprosthetic infection by clinical symptoms and histological examinations of surgical specimens. Two patients with TKA had a sinus tract communicating with the loose prosthesis. In the other patient with THA, purulent fluid was repeatedly aspirated but no organism grew on culture. All patients had pain in the affected joint with elevation of serum CRP and synovial white blood cell count. Histopathological examination including frozen and permanent sections were positive (more than five polymorphonuclear leukocytes per high-power field (Tsaras et al. 2012)) in all patients.

First surgical procedure consisted of implant removal, irrigation, and meticulous debridement. At the end of the first procedure, articulating antibiotic-impregnated cement spacer was placed in all patients. After the first procedure, all patients were treated with intravenous antibiotics at least 6 weeks. The second-stage revision arthroplasty was considered when there were no signs of recurrent infections after cessation of antibiotic therapy. According to Greidanus et al. (Greidanus et al. 2007), ESR $<22.5 \mathrm{~mm} / \mathrm{hr}$ and $\mathrm{CRP}<1.35 \mathrm{mg} / \mathrm{dl}$ were considered negative for infection. In addition to these laboratory tests and joint aspirations, the TPBS was routinely evaluated before any surgical procedures including debridement, cement spacer exchange, and second-stage revision arthroplasty. The final decision to perform the second-stage revision arthroplasty was based on the results of frozen section in addition to intraoperative gross findings. A frozen section was considered positive for infection if there were more than five polymorphonuclear leukocytes per high-power field in at least five separate microscopic fields (Tsaras et al. 2012). If the results of intraoperative frozen section were positive or purulent fluid was observed during surgery, thorough debridement and cement spacer exchange were repeated. Paraffin-embedded permanent sections were prepared following every surgery. A permanent section was considered positive for infection if there were more than five polymorphonuclear leukocytes per high-power field in at least five separate microscopic fields. The average number of surgical procedures required until the second-stage revision arthroplasty was 2.4 times (1-4 times) and the average treatment period was 4.8 months (3-13 months). Patients were advised to use wheelchair during the intervals between the first procedure and the revision arthroplasty. The mean follow-up after the revision arthroplasty was 3.2 years (range 2-7 years). To be judged infection-free at follow-up, a patient had to be free of clinical signs for infection (fever, local pain, redness, warmth, sinus tract infection) and have a normal CRP level and no radiological sign of osteolysis (Fink et al. 2009).

The TPBS was performed at a mean of 12 weeks (8-20 weeks) after the previous operation. The bone scintigraphy images were obtained after injecting technetium-99m-labelled diphosphonate $(555 \mathrm{mBq})$ and using GCA-7200 (Toshiba Medical Systems Corporation, Tochigi, Japan). The TPBS consisted of three phases (Nagoya et al. 2008); the blood flow phase, reflecting hyperaemia, was immediately after infusion of the tracer; the blood pool phase, reflecting inflammation, was between 3 and 5 minutes later, and the late phase, reflecting bone activity, between 3 and 4 hours after infusion. The TPBS images were transferred to a workstation (RS 6000, Intelli Station E Pro, IBM, USA) equipped with a 1,024 × 768 pixel solution monitor (IBM Co., Tokyo, Japan). A square region of interest of $5 \times 5$ pixels was placed over the most intense focus. Although scintigraphy has poor spatial resolution, it 
was possible to localize major anatomical structures. The accumulation of isotope within the region of interest in question was quantified using CIS-image (IBM Co., Tokyo, Japan). According to Nagoya et al. (Nagoya et al. 2008), the accumulation in the hip was expressed as a percentage compared with the gradation in the contralateral femoral artery for the blood flow phase, the femoral vein for the blood pool phase, and the anterior iliac crest for the late phase. Increased uptake of the isotope in the blood flow and blood pool phases was noted when the density was $\geqq 75 \%$ of the gradation in the femoral artery and femoral vein, respectively. Increased uptake in the late phases was noted when the density was equivalent to, or greater than, $90 \%$ of gradation in the anterior iliac crest. Similarly, the accumulation in the knee was compared with the gradation in the contralateral popliteal artery for the blood flow phase, the popliteal vein for the blood pool phase, and the tibial tuberosity for the late phase. Increased uptake in all three phases of TPBS was considered positive for infection (Nagoya et al. 2008). For assessing the intra- and inter-observer agreement of measurements, two observers performed the measurements twice, one week apart.

\section{Statistical analysis}

Histopathological results in addition to microbiologic examinations of surgical specimens represented the reference standard in all patients. Although the final decision to perform the second-stage revision arthroplasty was based on the frozen section results, the final histopathological diagnoses were made using permanent sections. Sensitivity, specificity, positive predictive value and negative predictive value for the TPBS in the diagnosis of infection were calculated. Intra- and inter-observer agreements were assessed using the interclass correlation coefficients (ICC). All analyses were performed by using a statistical software program (SPSS, version 11.5; SPSS, Chicago, IL).

\section{Results}

There was no recurrence of infection after the revision arthroplasty except 2 patients. Recurrent infection was noted 1 year after the revision THA in one patient and 2 years after the revision TKA in another patient. The patient with infected revision THA underwent delayed reimplantation surgery again. Another patient with infected revision TKA underwent arthrodesis of the knee because of soft tissue problems. There was no recurrence of infection in these two patients (2 and 3 year follow-up respectively).

There was no discordance between the results of frozen and permanent sections. Histopathological examination showed positive in 17 and negative in 13 out of 30 examinations. Among 17 samples positive for histopathology, there were only 4 samples positive for bacterial culture. All samples negative for histopathology showed negative for bacterial culture. Figure 1 shows the relationship between the results of TPBS and histopathological examination. The positive and negative predictive values for the presence of infection were $80 \%$ and $90 \%$, respectively. The diagnostic sensitivity was $94 \%$ and the specificity was $69 \%$. Figure 2 shows a patient without infection around the cement spacer of the right hip. The late phase was positive, while the blood flow and blood pool phases were negative. Figure 3 shows a patient with residual infection around the anitibiotic-impregnated cement spacer of the left knee, which was confirmed by intraoperative frozen section. The TPBS was positive in all three phases.

The inter-observer ICC was 0.82 and the intraobserver ICC was 0.88 , indicating good intra- and interobserver agreement on the TPBS measurements.

\section{Discussion}

There is a need to establish additional diagnostic tests for residual infection prior to the second-stage revision. To the best of our knowledge, this is the first study to evaluate the diagnostic value of TPBS for the diagnosis of infection around antibiotic-impregnated cement spacers. Based on our results, the diagnostic sensitivity of TPBS was $94 \%$ and the specificity was $69 \%$. Although the specificity was lower than the previous report on infected hip prosthesis (Nagoya et al. 2008), the high negative predictive value (90\%) makes the TPBS a clinically useful test. When the

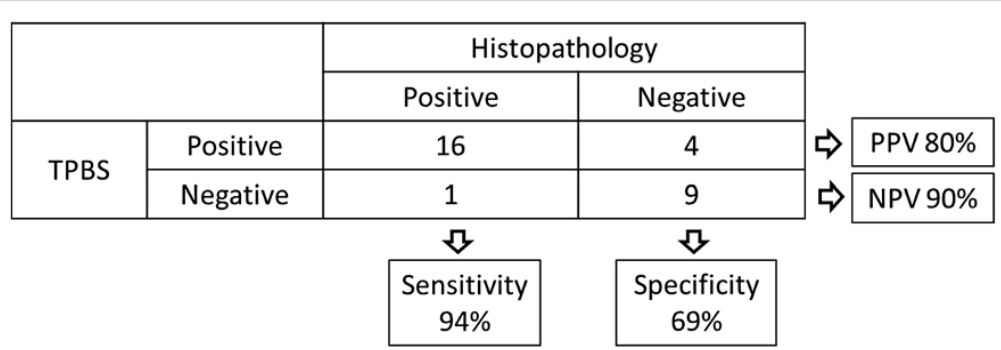

Figure 1 Relationship between the results of triple-phase bone scintigraphy and histopathological examination. TPBS: Triple-phase bone scintigraphy, PPV: positive predictive value, NPV: negative predictive value. 


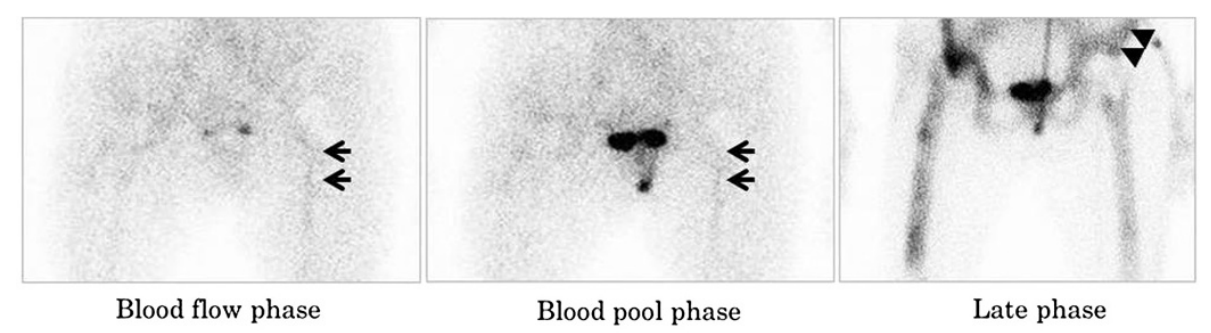

Figure 2 Triple-phase bone scintigraphy in a patient without infection around the cement spacer of the hip. Arrows indicate the contralateral femoral artery in the blood flow phase and the femoral vein in the blood pool phase. Arrowheads indicate the contralateral anterior iliac spine.

TPBS are negative, the residual infection around the cement spacer is unlikely. This information is particularly useful in patients with underlying inflammatory disease or malignancy, because CRP and ESR levels often fluctuate. In contrast, positive TPBS requires meticulous examinations including repeated aspirations and new laboratory markers such as interleukin-6, procalcitonin, and TNFalpha (Bottner et al. 2007).

Infection around the cement spacer induces inflammatory responses such as neovascularization and granulation formation. This condition is equivalent to septic loosening of prosthesis, which can be diagnosed using TPBS. Therefore, TPBS was useful in detecting residual infection around cement spacers. However, the first two phases of TPBS reflecting hyperemia and inflammation are not necessarily related to infection. It was expected that main factor affecting the results is postoperative period. In this study, TPBS was performed at least 8 weeks after the previous surgery. Although postoperative acute inflammation is considered to attenuate within the period of time, it is possible that longer intervals between previous surgery and TPBS could reduce falsepositive cases.

Recently, FDG-PET has been successfully used to detect periprosthetic infection (Zhuang et al. 2001; Delank et al. 2006; Zoccali et al. 2009; Mayer-Wagner et al. 2010). It is reported that FDG-PET is a more accurate diagnostic test than TPBS (Reinartz et al. 2005; Reinartz 2009). A systematic review reported that accuracy of FDG-PET was $89 \%$ for hip and $83 \%$ for knee arthroplasty, while TPBS was $80 \%$ for hip and $81 \%$ for knee (Reinartz 2009). In addition, Huang et al. (Huang et al. 2011) recently reported that FDG-PET was a feasible tool to help in detecting infection around antibiotic-loaded cement spacers. Although PET is highly effective procedure for detecting infection around prosthesis and cement spacers, its limitations are the restricted availability and the costs. TPBS yields a slightly lower accuracy, but excels in simplicity and cost-effectiveness (Reinartz 2009). Because FDGPET is not yet common, we believe that TPBS still has an important role in diagnosis of residual infection around prosthesis and cement spacers.

There are several limitations in this study. Firstly, this is a retrospective study with a relatively small number of patients during a 5-year period. Larger prospective studies are needed to corroborate these findings. Secondly, histopathological examination was used as the reference standard for the diagnosis of infection. At the final follow-up, recurrent infection was noted in two patients despite negative results in histopathology. Although

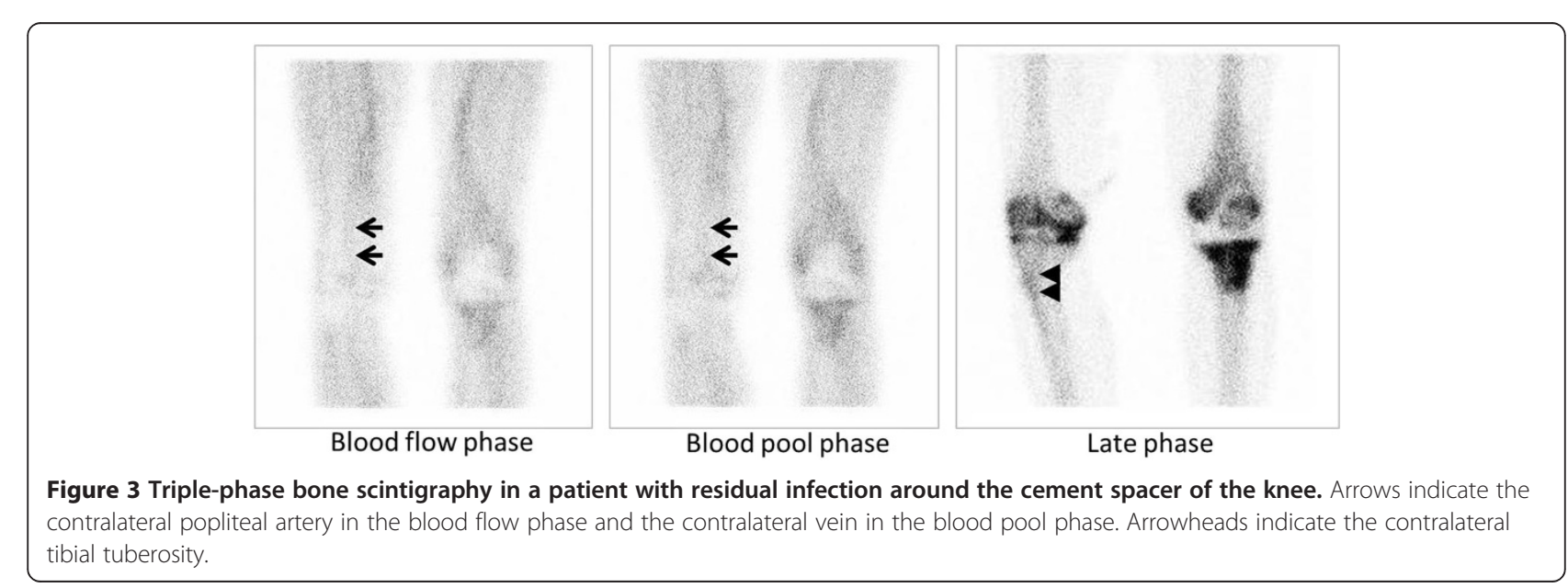


infection probably occurred long after the revision surgery in these two patients, we cannot exclude the possibility of the presence of infection at the revision surgery. Lastly, hips and knees were simultaneously evaluated in this study. Some authors reported that accuracy of FDGPET is lower in knee than in hip prosthesis (Zhuang et al. 2001; Reinartz 2009). Although it is reported that TPBS is less affected by the complexity of the knee joint (Reinartz 2009), the results might differ between hips and knees. We did not compare TPBS results between hips and knees because the number of patients with infected knees was quite small.

In conclusion, TPBS was useful in the diagnosis of infection around the articulating cement spacer. In particular, the high negative predictive value makes the TPBS a clinically useful test. When the TPBS are negative, the residual infection around the cement spacer is unlikely. We believe that TPBS is particularly useful in patients with underlying inflammatory disease or malignancy, because serological examinations such as CRP and ESR are not always reliable in the diagnosis of residual infection.

No benefits in any form have been received or will be received from a commercial party related directly or indirectly to the subject of this article.

\section{Consent}

Written informed consent was obtained from the patient for the publication of this report and any accompanying images.

\section{Competing interests}

The authors declare that they have no competing interests.

\section{Authors' contributions}

YO and MI participated in the design of the study and carried out measurements, data collection, and statistical analysis. MI, GF, KA, NS, TK, TT participated the design of the study and helped to draft the manuscript. All authors read and approved the final manuscript.

\section{Acknowledgements}

The authors are grateful to all the patients, physicians, and co-medical staffs who participated in this study.

\section{Author details}

'Department of Orthopaedic Surgery, Kochi Medical School, Kochi University, Kohasu, Oko-cho, Nankoku, Kochi 783-8505, Japan. ${ }^{2}$ School of Health Science and Social Welfare, Kibi International University, Takahashi, Okayama 720-0001, Japan.

Received: 27 June 2013 Accepted: 21 August 2013

Published: 27 August 2013

\section{References}

Bare J, MacDonald SJ, Bourne RB (2006) Preoperative evaluations in revision total knee arthroplasty. Clin Orthop Relat Res 446:40-44

Bauer TW, Parvizi J, Kobayashi N, Krebs V (2006) Diagnosis of periprosthetic infection. J Bone Joint Surg Am 88:869-882

Berbari E, Mabry T, Tsaras G, Spangehl M, Erwin PJ, Murad MH, Steckelberg J, Osmon D (2010) Inflammatory blood laboratory levels as markers of prosthetic joint infection: a systematic review and meta-analysis. J Bone Joint Surg Am 92:2102-2109
Biring GS, Kostamo T, Garbuz DS, Masri BA, Duncan CP (2009) Two-stage revision arthroplasty of the hip for infection using an interim articulated Prostalac hip spacer: a 10- to 15-year follow-up study. J Bone Joint Surg Br 91:1431-1437

Borowski M, Kusz D, Wojciechowski P, Cielinski L (2012) Treatment for periprosthetic infection with two-stage revision arthroplasty with a gentamicin loaded spacer. The clinical outcomes. Ortop Traumatol Rehabil 14:41-54

Bottner F, Wegner A, Winkelmann W, Becker K, Erren M, Gotze C (2007) Interleukin-6, procalcitonin and TNF-alpha: markers of peri-prosthetic infection following total joint replacement. J Bone Joint Surg Br 89:94-99

Cui Q, Mihalko WM, Shields JS, Ries M, Saleh KJ (2007) Antibiotic-impregnated cement spacers for the treatment of infection associated with total hip or knee arthroplasty. J Bone Joint Surg Am 89:871-882

Delank KS, Schmidt M, Michael JW, Dietlein M, Schicha H, Eysel P (2006) The implications of 18F-FDG PET for the diagnosis of endoprosthetic loosening and infection in hip and knee arthroplasty: results from a prospective, blinded study. BMC Musculoskelet Disord 7:20

Fehring TK, Odum S, Calton TF, Mason JB (2000) Articulating versus static spacers in revision total knee arthroplasty for sepsis. The Ranawat Award. Clin Orthop Relat Res 380:9-16

Fink B, Grossmann A, Fuerst M, Schafer P, Frommelt L (2009) Two-stage cementless revision of infected hip endoprostheses. Clin Orthop Relat Res 467:1848-1858

Garvin KL, Konigsberg BS (2011) Infection following total knee arthroplasty: prevention and management. J Bone Joint Surg Am 93:1 167-1175

Greidanus NV, Masri BA, Garbuz DS, Wilson SD, McAlinden MG, Xu M, Duncan CP (2007) Use of erythrocyte sedimentation rate and C-reactive protein level to diagnose infection before revision total knee arthroplasty. A prospective evaluation. J Bone Joint Surg Am 89:1409-1416

Huang MJ, Hsieh PH, Ueng SW, Ho KC, Yen TC, Lee MS (2011) Use of positron emission tomography to detect infection around antibiotic-loaded cement spacers in patients with high C-reactive protein levels. Orthopedics 34:e605-609

Mahmud T, Lyons MC, Naudie DD, Macdonald SJ, McCalden RW (2012) Assessing the gold standard: a review of 253 two-stage revisions for infected TKA. Clin Orthop Relat Res 470:2730-2736

Mayer-Wagner S, Mayer W, Maegerlein S, Linke R, Jansson V, Muller PE (2010) Use of 18F-FDG-PET in the diagnosis of endoprosthetic loosening of knee and hip implants. Arch Orthop Trauma Surg 130:1231-1238

Nagoya S, Kaya M, Sasaki M, Tateda K, Yamashita T (2008) Diagnosis of periprosthetic infection at the hip using triple-phase bone scintigraphy. J Bone Joint Surg Br 90:140-144

Pedersen AB, Svendsson JE, Johnsen SP, Riis A, Overgaard S (2010) Risk factors for revision due to infection after primary total hip arthroplasty. A populationbased study of 80,756 primary procedures in the Danish Hip Arthroplasty Registry. Acta Orthop 81:542-547

Phillips JE, Crane TP, Noy M, Elliott TS, Grimer RJ (2006) The incidence of deep prosthetic infections in a specialist orthopaedic hospital: a 15-year prospective survey. J Bone Joint Surg Br 88:943-948

Reinartz P (2009) FDG-PET in patients with painful hip and knee arthroplasty: technical breakthrough or just more of the same. Q I Nucl Med Mol Imaging 53:41-50

Reinartz P, Mumme T, Hermanns B, Cremerius U, Wirtz DC, Schaefer WM Niethard F, Buell U (2005) Radionuclide imaging of the painful hip arthroplasty: positron-emission tomography versus triple-phase bone scanning. J Bone Joint Surg Br 87:465-470

Rubello D, Borsato N, Chierichetti F, Zanco P, Ferlin G (1995) Three-phase bone scintigraphy pattern of loosening in uncemented hip prostheses. Eur J Nucl Med 22:299-301

Sanzen L, Sundberg M (1997) Periprosthetic low-grade hip infections. Erythrocyte sedimentation rate and C-reactive protein in 23 cases. Acta Orthop Scand 68:461-465

Schauwecker DS (1992) The scintigraphic diagnosis of osteomyelitis. AJR Am J Roentgenol 158:9-18

Silva M, Tharani R, Schmalzried TP (2002) Results of direct exchange or debridement of the infected total knee arthroplasty. Clin Orthop Relat Res 404:125-131

Stumpe KD, Notzli HP, Zanetti M, Kamel EM, Hany TF, Gorres GW, von Schulthess GK, Hodler J (2004) FDG PET for differentiation of infection and aseptic loosening in total hip replacements: comparison with conventional radiography and three-phase bone scintigraphy. Radiology 231:333-341

Sukeik M, Haddad FS (2009) Two-stage procedure in the treatment of late chronic hip infections-spacer implantation. Int J Med Sci 6:253-257 
Tsaras G, Maduka-Ezeh A, Inwards CY, Mabry T, Erwin PJ, Murad MH, Montori VM, West CP, Osmon DR, Berbari EF (2012) Utility of intraoperative frozen section histopathology in the diagnosis of periprosthetic joint infection: a systematic review and meta-analysis. J Bone Joint Surg Am 94:1700-1711

Tunney MM, Patrick S, Curran MD, Ramage G, Hanna D, Nixon JR, Gorman SP, Davis Rl, Anderson N (1999) Detection of prosthetic hip infection at revision arthroplasty by immunofluorescence microscopy and PCR amplification of the bacterial 16S rRNA gene. J Clin Microbiol 37:3281-3290

Ure K, Amstutz HC, Nasser S, Schmalzried TP (1998) Direct-exchange arthroplasty for the treatment of infection after total hip replacement. An average ten-year follow-up. J Bone Joint Surg Am 80:961-968

Van Kleunen JP, Knox D, Garino JP, Lee GC (2010) Irrigation and debridement and prosthesis retention for treating acute periprosthetic infections. Clin Orthop Relat Res 468:2024-2028

Zhuang H, Duarte PS, Pourdehnad M, Maes A, Van Acker F, Shnier D, Garino JP, Fitzgerald RH, Alavi A (2001) The promising role of 18F-FDG PET in detecting infected lower limb prosthesis implants. J Nucl Med 42:44-48

Zoccali C, Teori G, Salducca N (2009) The role of FDG-PET in distinguishing between septic and aseptic loosening in hip prosthesis: a review of literature. Int Orthop 33:1-5

doi:10.1186/2193-1801-2-401

Cite this article as: Ikeuchi et al: Diagnostic value of triple-phase bone scintigraphy for the diagnosis of infection around antibioticimpregnated cement spacers. SpringerPlus 2013 2:401.

\section{Submit your manuscript to a SpringerOpen ${ }^{\circ}$ journal and benefit from:}

- Convenient online submission

- Rigorous peer review

- Immediate publication on acceptance

- Open access: articles freely available online

- High visibility within the field

- Retaining the copyright to your article

Submit your next manuscript at $\gg$ springeropen.com 